\title{
Quantum anomalous Hall effect in graphene coupled to skyrmions
}

\author{
J. L. Lado ${ }^{1}$ and J. Fernández-Rossier ${ }^{1,2}$ \\ ${ }^{1}$ International Iberian Nanotechnology Laboratory (INL), Avenida Mestre José Veiga, 4715-330 Braga, Portugal \\ ${ }^{2}$ Departamento de Fisica Aplicada, Universidad de Alicante, San Vicente del Raspeig, Alicante E-03690, Spain
}

(Received 11 May 2015; published 21 September 2015)

\begin{abstract}
Skyrmions are topologically protected spin textures, characterized by a topological winding number $N$, that occur spontaneously in some magnetic materials. Recent experiments have demonstrated the capability to grow graphene on top Fe/Ir, a system that exhibits a two-dimensional skyrmion lattice. Here we show that a weak exchange coupling between the Dirac electrons in graphene and a two-dimensional skyrmion lattice with $N= \pm 1$ drives graphene into a quantum anomalous Hall phase, with a band gap in bulk, a Chern number $\mathcal{C}=2 N$, and chiral edge states with perfect quantization of conductance $G=2 N \frac{e^{2}}{h}$. Our findings imply that the topological properties of the skyrmion lattice can be imprinted in the Dirac electrons of graphene.
\end{abstract}

DOI: 10.1103/PhysRevB.92.115433

PACS number(s): 73.22.Pr, 73.43.Cd, 75.70.Kw

\section{INTRODUCTION}

Graphene is a zero gap semiconductor on the brink of becoming a topological insulator. It is no coincidence that several predictions of topological phases in two-dimensional systems are based on some small modification of the model that actually describes graphene, namely, a tight-binding Hamiltonian for electrons in a honeycomb lattice at half filling. In a seminal paper [1], Haldane proposed that a honeycomb crystal with a suitable magnetic flux decoration would become an insulator with a quantized Hall response, without Landau levels. The ground-breaking proposal of the quantum spin Hall (QSH) phase by Kane and Mele [2,3], a topological insulating phase with time reversal, is also based on graphene with spin-orbit coupling, that is mathematically related to the Haldane model. A QSH-like phase was also predicted for graphene under the influence of a perpendicular magnetic field and ferromagnetic order [4]. In addition, the quantum Hall effect has been observed in graphene at a relatively low magnetic field at low temperatures [5], and even at room temperature [6] at high fields. Furthermore, the combination of exchange interaction and spin-orbit coupling has been predicted to give rise to the QAH phase [7]. Here we propose a topological phase that, unlike all of the above, requires no spin-orbit coupling and no applied magnetic field.

Topological insulating phases in two dimensions attract interest because of the very special transport properties, such as the perfect conductance quantization of the quantum Hall phase [8]. These special transport properties arise from the fact that topological phases have a gapped bulk and conducting edge states. Interestingly, QAH phases have chiral edge states for which intra-edge backscattering is impossible. Thus, quantization of conductance of the QAH phase should be as perfect as the one observed in the quantum Hall effect. This contrasts with QSH edge states, for which only the total absence of time-reversal symmetry breaking impurities, such as nuclear spins or local moments, prevents backscattering. As a result, the quantization of conductance in QSH and QSH-like phases is far [5,9] from the perfection observed in graphene QH systems [6,8].

The notion that exchange interaction with the local moments with noncollinear spin textures affects severely the kinetic energy of itinerant electrons goes back to the proposal of the double exchange mechanism [10]. Later, the role of nontrivial effects on the Berry phase was recognized [11,12], and the notion that noncollinear spin textures induce an effective orbital magnetic field that would lead to an anomalous Hall term was put forward [13-19]. It was also shown by Ohgushi et al. [20] that the double exchange model on a two-dimensional kagome lattice with noncollinear classical spins led to a quantum anomalous Hall phase with quantized Hall conductance. Recent experiments have demonstrated the possibility of growing graphene islands on top of a twodimensional skyrmion lattice hosted by a single atomic layer of Fe deposited on an $\operatorname{Ir}(111)$ substrate [21]. This experiment, together with the notion that noncollinear spin textures induce very interesting effects on conduction electrons [11-13,1820], motivates our study of graphene electrons coupled to a skyrmion lattice. Interestingly, we find that this system can exhibit the quantum anomalous Hall effect [Figs. 1(e) and 1(f)], a topological phase of matter that is being actively pursued in condensed-matter physics [22].

The paper is organized as follows. In Sec. II we present the tight-binding model to study the skyrmion proximity effect in graphene. In Sec. III we show the nontrivial phases that arise by proximizing graphene to a triangular and rectangular skyrmion lattice, respectively, in the weak-coupling limit at half filling. Finally, in Sec. IV we summarize our conclusions.

\section{MODEL}

Skyrmions are noncollinear spin textures [Figs. 1(a) and 1(b)] characterized by a topological number [23]

$$
N=\frac{1}{4 \pi} \int \vec{n} \cdot\left(\frac{\partial \vec{n}}{\partial x} \times \frac{\partial \vec{n}}{\partial y}\right) d A,
$$

where $\vec{n}=\frac{\vec{M}}{|\vec{M}|}$ is the unit vector associated to the skyrmion magnetization. Skyrmions are being very actively studied in the context of spintronics [23,24], and they have been found both in bulk compounds [23] and two-dimensional systems [25]. Here we propose a mechanism to induce the QAH phase in graphene, fully independent of the spin-orbit coupling of carbon. Namely, it is based on exchange interaction with a magnetically ordered surface that hosts a skyrmion lattice [Fig. 1(a)]. 
(a)
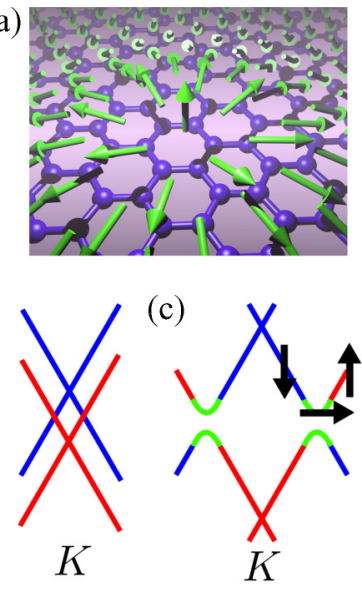

(e) $\quad C=0$

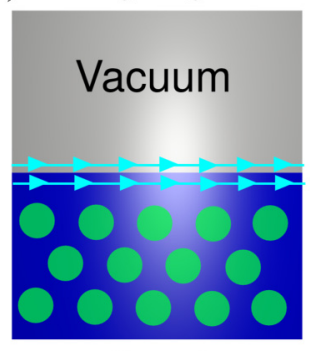

$C=+2$ (b)

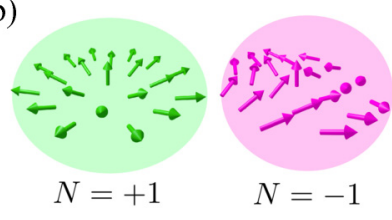

(d)

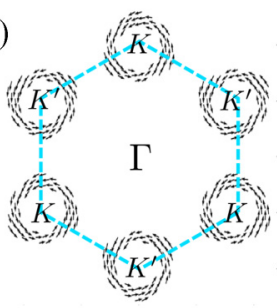

(f)

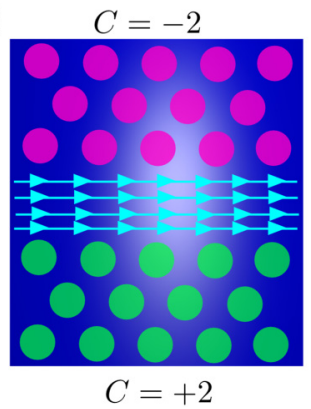

FIG. 1. (Color online) (a) Scheme of a graphene layer deposited on a skyrmion lattice (only the skyrmion cores are shown). (b) Skyrmions with $N= \pm 1$, that trigger a QAH. (c) Influence of the exchange to the skyrmions on the Dirac cones: spin splitting and band-gap opening. (d) Spin texture induced in the reciprocal space by the skyrmion driven gap opening. Edge states between vacuum and two-dimensional (2D) skyrmion crystal (e), and between two skyrmionic crystals with different chirality (f).

Our starting point is the tight-binding model for electrons in graphene, $\mathcal{H}_{0}=\sum_{i j \sigma} c_{j \sigma}^{\dagger} c_{i \sigma}$, plus their exchange to an arbitrary magnetization field, that is treated classically:

$$
\mathcal{H}=\mathcal{H}_{0}+J \sum_{i} \vec{S}_{i} \cdot \vec{M}_{i}
$$

Here $i$ labels the sites of the honeycomb lattice, $J$ is the short-range exchange interaction constant, $\vec{S}_{i}$ and $\vec{M}_{i}=M_{0} \vec{n}_{i}$ are the electronic spin-density operator and classical magnetization, respectively, evaluated at site $i$. The length of the magnetization field $M_{0}$ is assumed to be the same for all sites. Unlike most of the previous work [26], we focus on the weak-coupling limit $J \ll t$, adequate for proximity induced magnetism. Therefore, the exchange field acts as a perturbation on the Dirac spectrum.

The magnetization of a single skyrmion can be expressed as a map from the plane described in polar coordinates $r, \phi$ to the unit sphere, described in spherical coordinates $\Theta, \Phi$,

$$
\vec{n}=(\sin \Theta(r) \cos \Phi(\phi), \sin \Theta(r) \sin \Phi(\phi), \cos \Theta(r)),
$$

where $\Theta(r)$ is such that $\Theta(r=0)=0$ and $\Theta\left(r>R_{S k y}\right)=\pi$, where $R_{S k y}$ is the skyrmion radius. $\Phi(\phi)=N \phi+\gamma$, where $N$ is the skyrmion number that accounts for its vorticity [23] and $\gamma$ is a phase that determines the helicity of the skyrmion.

Since $\gamma$ can be gauged by a rotation along the $z$ axis, we take $\gamma=0$ without loss of generality. For the sake of simplicity, in the following we will assume a stepwise profile for the magnetization,

$$
\Theta^{\text {hard }}(r)= \begin{cases}0, & r=0, \\ \pi / 2, & 0<r<R_{S k y}, \\ \pi, & r>R_{S k y} .\end{cases}
$$

We have verified that the results do not change qualitatively if we use a smooth parametrization of the azimuthal angle.

\section{QUANTUM ANOMALOUS PHASE AT WEAK COUPLING}

In this section we present the result for the weak exchange limit $(J \ll t)$. This situation is the one to be realistically obtained for graphene on top of a skyrmion lattice. We show that a topological gap opens for arbitrarily small exchange coupling and independently on the type of skyrmion lattice, triangular or rectangular.

\section{A. Triangular skyrmion lattice}

We now consider the properties of graphene interacting with a triangular crystal of skyrmions. In Fig. 2(b) we show the energy bands for a $5 \times 5$ supercell with one skyrmion. It is apparent that exchange interaction with the skyrmions opens up a gap in graphene. This can be understood as follows. We write the exchange part of the Hamiltonian as

$$
\mathcal{V}=+J \sum_{I} \vec{S}_{I} \cdot\left(\langle\vec{M}\rangle+\delta \vec{M}_{I}\right),
$$

where $\langle\vec{M}\rangle=\frac{1}{N} \sum_{I} \vec{M}_{I}$ is the average magnetization, and $\delta \vec{M}_{I}=\vec{M}_{i}-\langle\vec{M}\rangle$ are the fluctuations. Ignoring the fluctuation term, the average magnetization induces a spin splitting, shown in Fig. 1(b), where the bands have a well defined spin along the average magnetization. The resulting conduction band of one spin projection is degenerate with the valence band of the opposite spin projection, defining a circle of degenerate points that, at half filling, happens to be the Fermi surface [see Fig. 1(c)]. For noncollinear spin textures, $\delta \vec{M}$ has terms orthogonal to $\langle\vec{M}\rangle, \delta \vec{M}_{\perp}$, that open up the gap at the degeneracy circle, and creates a spin vorticity in the reciprocal space [see Fig. 1(d)]. A similar argument has been used by Qiao and co-workers in their proposal [7] for QAH in graphene with Rashba spin-orbit coupling and an exchange field. In our system the $\delta \vec{M}_{\perp}$ part of the Hamiltonian plays the same role as the Rashba coupling in their model. However, we found that only skyrmions with topological charge $N= \pm 1$ are able to open a topological gap.

The nontrivial nature of the gap of Fig. 2(b) can be anticipated from the Berry curvature [27] profile shown in Fig. 2(d). A nonvanishing Berry curvature arises at the crossing circle of the spin-split Dirac points [see Fig. 2(c)], where the spin-flip terms open up a gap.

The Hall conductivity can be be expressed as an integral of the Berry curvature $[1,18,28]$ :

$$
\sigma_{x y}\left(E_{F}\right)=\frac{1}{2 \pi} \frac{e^{2}}{h} \sum_{n} \int f\left(\epsilon_{n}(\vec{k}), E_{F}\right) \Omega_{n}(\vec{k}) d^{2} k,
$$


(a)
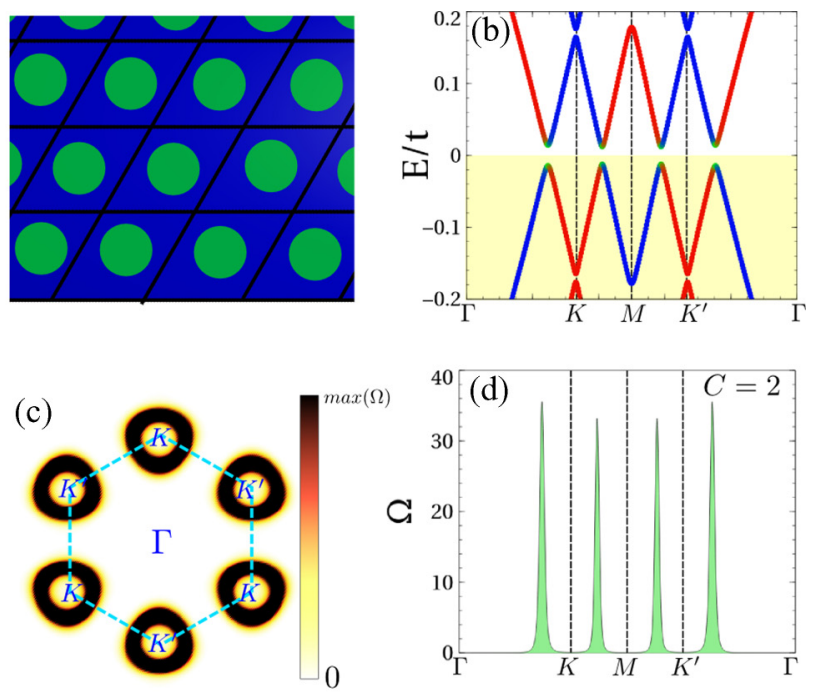

(e)
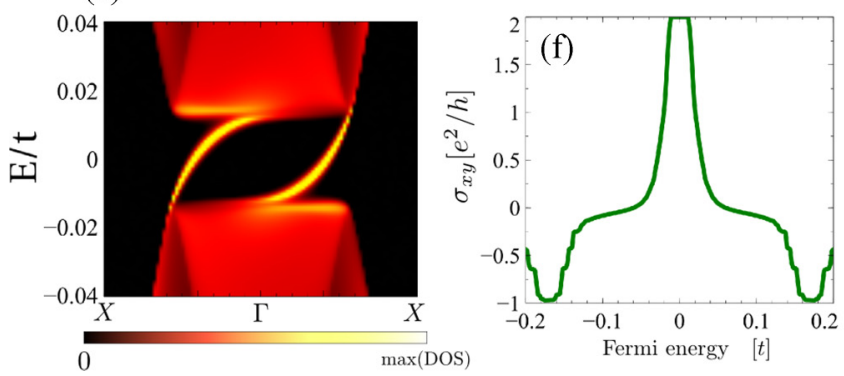

FIG. 2. (Color online) (a) Scheme of a triangular arrangement of skyrmions and (b) band structure of a $5 \times 5$ honeycomb supercell with a skyrmion with winding number equal to 1 and $J=0.3 t$. (c) Contour plot of the Berry curvature, with a small trigonal warping around the corners of the hexagonal Brillouin zone. (d) Positive Berry curvature localized at the region of band-gap opening. (e) Surface density of states on the termination of a semi-infinite graphene plane, showing two in-gap chiral states. (f) Anomalous Hall conductivity as a function of Fermi energy.

where $f\left(\epsilon_{n}(\vec{k})\right)$ is the Fermi occupation function, and $n$ labels the bands. When the Fermi energy $E_{F}$ lies inside a gap, the Hall conductivity is proportional to a Chern number $\mathcal{C}$ [28], which is an integer number $\mathcal{C}$, resulting in a quantized Hall conductivity $\sigma_{x y}=\mathcal{C} e^{2} / h$. Our calculations show that when $E_{F}$ lies in the gap opened by the coupling to the $N= \pm 1$ skyrmions, the Chern number is given by

$$
\mathcal{C}=2 N
$$

This is the central result of this work: the topological winding number of the skyrmions is imprinted into the Dirac electrons. Several consequences follow. First, two chiral edge states are expected to occur at the boundaries of the crystal. This is confirmed by our calculations, using a recursive Green-function method [29] to calculate the surface states, as shown in Fig. 2(e). Second, an interface between two skyrmion lattices, with opposite skyrmion numbers $N=1$ and $N=-1$, is expected to show four interface states according to the index theorem [see Fig. 1(f)]. Third, when $E_{F}$ lies inside the gap, the Hall conductance is quantized $[18,28]$, (a)

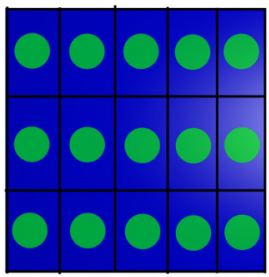

(c)
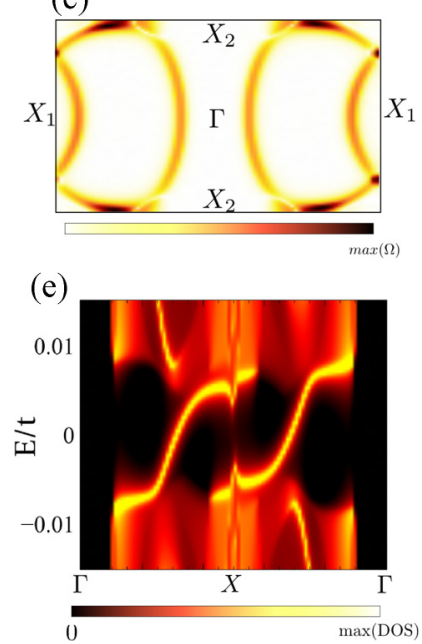
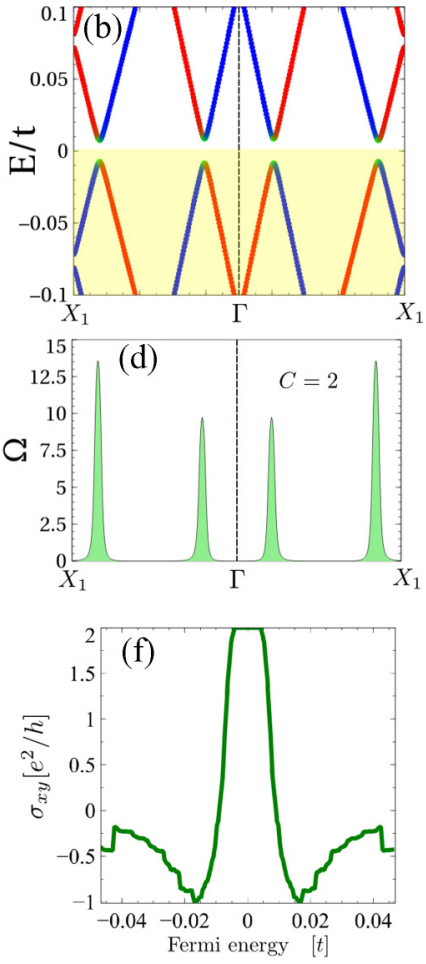

FIG. 3. (Color online) (a) Scheme of a rectangular skyrmion crystal. (b) Band structure of half filled graphene with a rectangular unit cell, coupled to the rectangular skyrmion lattice with $J=0.3 t$. (c) Berry curvature in the whole Brillouin zone, showing a same sign behavior which sums up to a $\mathcal{C}=+2$ total Chern number. (d) Berry curvature along the $k$ path shown in the band structure, showing a nonvanishing contribution in the anticrossings points. (e) Surface DOS in a semi-infinite geometry showing two chiral states. (f) Anomalous Hall conductivity as a function of Fermi energy.

$\sigma_{x y}=\mathcal{C} \frac{e^{2}}{h}$. Importantly, the Hall conductivity also takes large, but not-quantized, values when the Fermi energy lies close to, but outside, the gap [see Figs. 2(f) and 3(f)], on account of the finite Berry curvature integrated over the occupied states [30].

\section{B. Rectangular skyrmion lattice}

We now address the question of the influence of the type of skyrmion lattice on the existence of the QAH phase. In particular, motivated by experimental results [21], we consider a rectangular skyrmionic crystal, commensurate with the graphene lattice [see Fig. 3(a)]. We find that the topological character of such a system strongly depends on whether the valley mixing is an important effect.

The topological phase is observed for those unit cells in which the two valleys fold to different points in the Brillouin zone, which avoid intervalley mixing. As in the triangular case, the in-plane components open up a gap in the exchange split bands [Fig. 3(b)], which leads to a finite nonvanishing Berry curvature localized in the band crossing points [Fig. 3(d)]. The Fermi surface in this case can be more complex than in the triangular skyrmion lattice due to the large folding of the reciprocal space, which is also reflected in Berry curvature, Fig. 3(c). The calculated Chern number is also $\mathcal{C}=2 N$, as in 
the triangular lattice, which in a semi-infinite geometry gives rise to two copropagating edge branches [Fig. 3(e)].

Away from half filling, when the Fermi energy no longer lies within the gap, the anomalous response is still nonvanishing up to energies four times the gap [Fig. 3(f)]. The sign of the anomalous response is again the same for electrons and holes as in the triangular case. In comparison, upon entering the conduction (valence) band, the response can rapidly become negative and with a value close to 1 , due to the presence of an edge branch located in the valence (conduction) band [see Fig. 3(e)] that coexists with normal valence (conduction) states.

\section{Experimental verification}

The experimental verification of our proposal is not far from the state of the art. The optimal conditions to detect perfect edge transport associated to the QAH phase is induced by a skyrmion lattice hosted by an insulating magnetic material that couples to the graphene electrons. Three ingredients have been demonstrated in different systems. First, recent experimental results show the possibility of growing graphene on the surface of Fe/Ir, a surface that hosts a skyrmion lattice [21]. Second, and independent from the first, recent experiments show that nonquantized anomalous Hall is induced in graphene by proximity to a ferromagnetic insulating substrate [31]. Finally, skyrmion lattices have been observed in insulating chiral-lattice magnet $\mathrm{Cu}_{2} \mathrm{OSeO}_{3}$ [32].

The critical figure of merit to realize our proposal is the magnitude of the skyrmion induced gap. This is controlled by the strength of the exchange field of the underlying magnetic state. To gain some insight of the gap opening mechanism, it is convenient to change independently the off-plane (exchange shift) and in-plane (spin mixing) components of the skyrmion texture. Our numerical calculations [see Fig. 4(b)] for the triangular lattice of hard-core skyrmions $\left[n_{z}\left(r<R_{\mathrm{Sky}}\right)=0\right]$
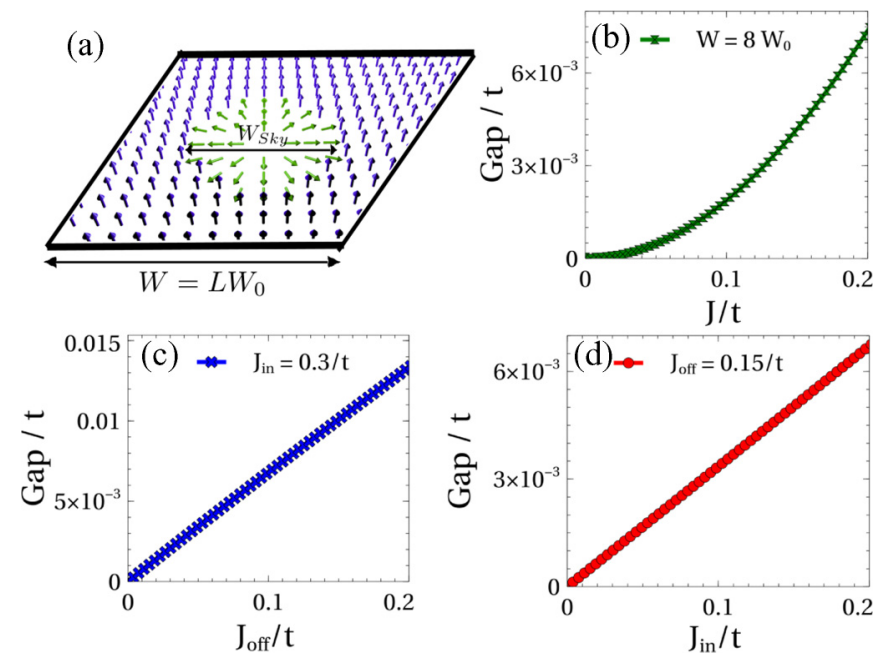

FIG. 4. (Color online) (a) Scheme of a hard-core skyrmion, showing an in-plane radial magnetic profile and an out-core off-plane magnetism. Evolution of the topological gap at half filling with the global exchange (b), only the out-core exchange (c), and the core exchange (d). show that the gap satisfies $\Delta \propto J_{\text {in }} J_{\text {off }} / t$ where $J_{\text {in }}$ and $J_{\text {off }}$ are the magnitude of the in-plane and off-plane components of the exchange field [see Figs. 4(b)-4(d)]. In the case of constant exchange strength, a quadratic exchange dependence is obtained, $\Delta \propto J^{2} / t$.

For reference, and given that there are no measurements of $J$, we take it from DFT calculations for graphene on top of $\mathrm{BiFeO}_{3}$ [33] that give $J=70 \mathrm{meV}$, and $t=2.6 \mathrm{eV}$. With the previous parameters, the topological gap would get a value of $\Delta \simeq 0.4 \mathrm{meV}$, within reach of transport spectroscopy. Taking these numbers, the window of $E_{F}$ within which the nonquantized intrinsic Hall effect would be sizable extends in a window of 1-3 meV around the Dirac energy. Our calculations indicate that the nonquantized Hall conductivity would be larger in the case of the rectangular lattice [Fig. 3(f)]. We also note that the sign of the skyrmion-induced Hall contribution would be the same for electrons and holes, in contrast with the conventional Hall effect.

\section{CONCLUSION}

We have shown that, at half filling, graphene with a weak exchange coupling to a skyrmion lattice develops a quantum anomalous Hall phase, with gapped bulk and chiral edge states that should have perfect quantization. This occurs at least for two different skyrmion lattices, rectangular and triangular, and seems a generic feature as long as the skyrmion lattice does not produce valley mixing. The Chern invariant $\mathcal{C}$ that characterizes the QAH phase is given by the topological invariant that describes the individual skyrmions $N$, through the remarkable relation $\mathcal{C}=2 N$, valid for $N= \pm 1$. Thus, graphene edges will have two chiral edge states, and graphene on top of an interface between two skyrmions lattices with opposite skyrmion number $N=+1$ and $N=-1$ will have four chiral edge states. Our proposal is different from previous proposals of QAH phase in graphene because it requires no spin-orbit coupling and no magnetic field acting on the graphene electrons.

Note added. During the final stages of the completion of this manuscript, we became aware of a work predicting a quantum anomalous phase for electrons on a square lattice coupled to skyrmions [26] in the strong-coupling limit $(J \gg t)$.

\section{ACKNOWLEDGMENTS}

J.F.R. acknowledges financial support by MEC-Spain (Grant No. FIS2013-47328-C2-2-P) and Generalitat Valenciana (Grant No. ACOMP/2010/070), Prometeo. This work has been financially supported in part by FEDER funds. We acknowledge financial support by Marie-Curie-ITN Grant No. 607904-SPINOGRAPH. J.L.L. acknowledges the hospitality of the Departamento de Fisica Aplicada at the Universidad de Alicante. We thank L. Brey and D. Jacob for useful discussions.

\section{APPENDIX A: STRONG-COUPLING LIMIT}

We discuss here the behavior of the system in the large- $J$ limit, where $J \gg t$ and the spin splitting of the bands is much larger than the bandwidth. This strong-coupling limit has been considered in previous works [20], but is not realistic in the 

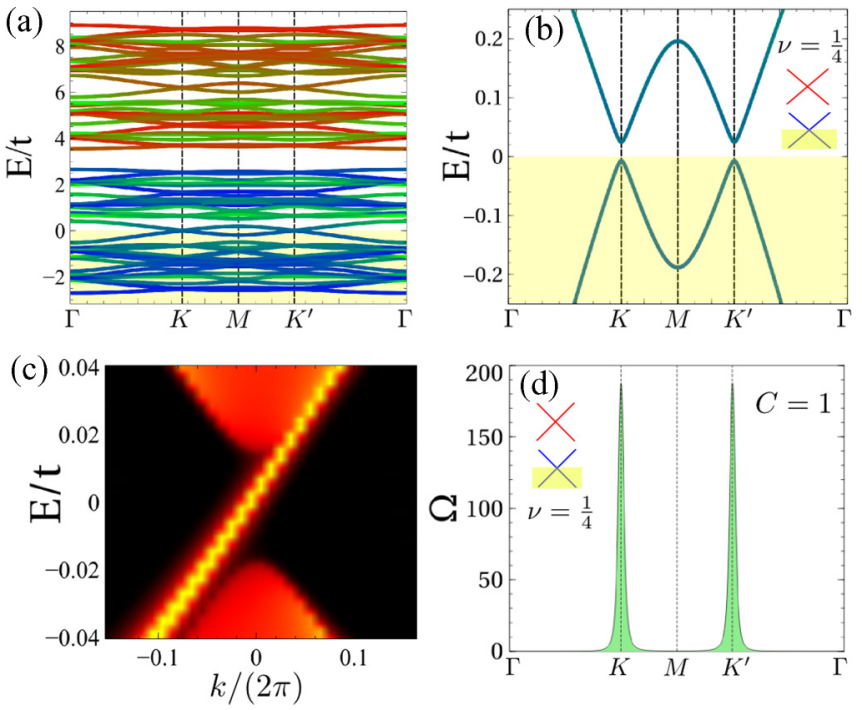

FIG. 5. (Color online) Graphene supercell over a triangular skyrmion crystal. Band structure (a), (b) at filling $1 / 4$ in the large exchange limit $(J=3 t)$. As shown by the surface density of states (c) and the Berry curvature (d), the system develops a quantum anomalous Hall state with a total Chern number $\mathcal{C}=1$. At large exchange, the half filling state becomes trivial, whereas the filling $1 / 4$ becomes topological resembling the Haldane model.

case of graphene. In the strong-coupling limit at half filling, the system behaves as a topologically trivial magnetic insulator, where both valence and conduction states of the majority spin are completely full. Since spin degeneracy is completely lifted in this case, the Fermi energy lies at the Dirac point at quarter filling. In this case (Fig. 5), the low-energy states are described by a gapped Dirac-like spectrum and our calculations in this limit give $\mathcal{C}=1$, for $N=1$. Therefore, this is topologically different than the weak-coupling case discussed in the main text, and much closer to the original QAH phase proposed by Haldane [1].

The physical origin of this topological phase can be understood as follows. The standard [13,18-20] spin rotation is performed, so that the exchange term is always diagonal $[12,13]$. The local nature of this transformation generates a coupling to a gauge field in the hopping operator [13,18-20] that describes an effective inhomogeneous magnetic field, that is responsible for the band-gap opening in this limit. In that limit, a skyrmion with topological number $N$ creates an effective field equal to $N \Phi_{0}$, where $\Phi_{0}=e / h$ is the magnetic flux quantum [23].

\section{APPENDIX B: TRIVIAL GAP OPENING}

In this section we will discuss some of the situations in which a skyrmion texture will not give rise to a topological gap. First we emphasize that strong defects or inhomogeneities in graphene, the underlying skyrmion lattice, or the exchange coupling can give rise to a phase transition between the topological state and a trivial state. This problem is common to any engineered topological state, and its study relies on the particular features of the microscopic models, which depend on the underlying material that hosts the skyrmions.
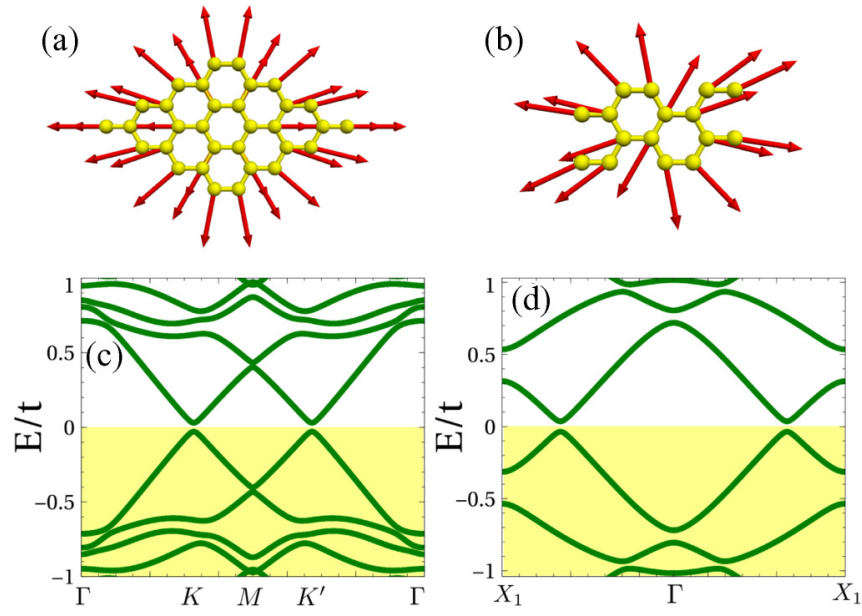

FIG. 6. (Color online) Unit cells (a), (b) and band structures (c), (d) for graphene over a fully in-plane triangular (a), (c) and rectangular (b), (d) skyrmion lattice. A gap is opened in the band structure, but the Chern number is identically zero, yielding a trivial insulator.

In the following we focus on homogeneous effects well captured by our phenomenological model that can ruin the perfect $\mathcal{C}=2$ state. We will discuss two situations: the case of a pure coplanar spin texture, and situations with strong intervalley mixing.
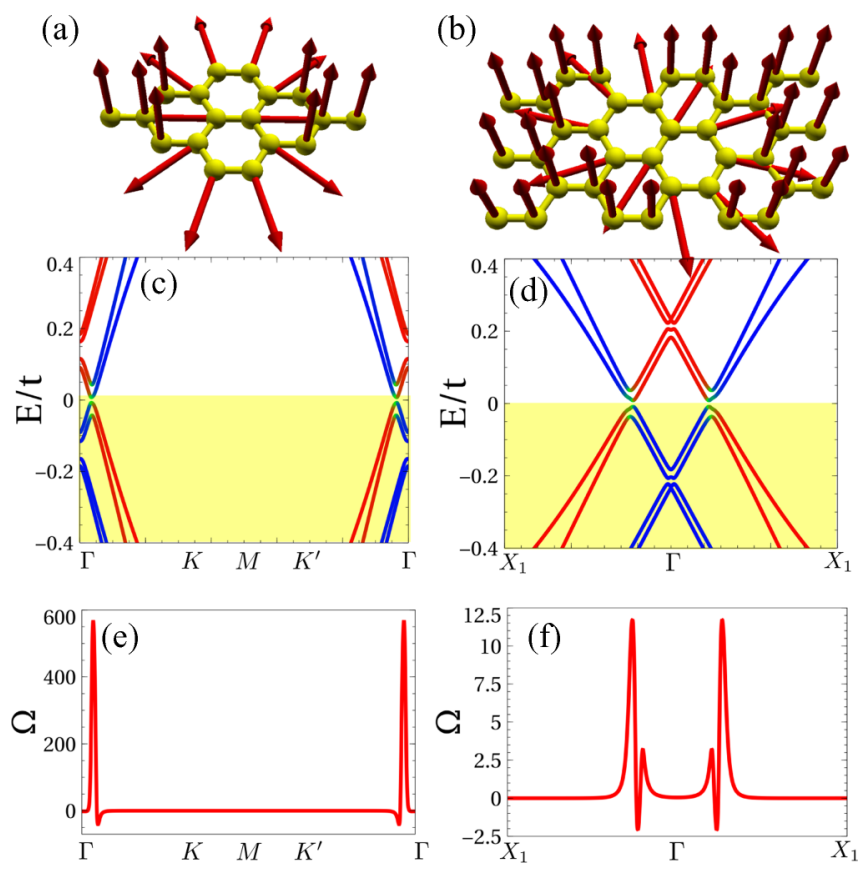

FIG. 7. (Color online) Unit cells (a), (b), band structures (c), (d), and Berry curvatures (e), (f) for graphene over a triangular (a), (c) and rectangular (b), (d) skyrmion lattice. The commensuration of graphene with the skyrmion lattice cause both valleys to be folded onto the $\Gamma$ point. Even though a gap with nonvanishing Berry curvature opens up, the sign changes along the Brillouin zone, and summing up all the contributions gives a vanishing Chern number. In the present situation, the gap is dominated by intervalley mixing. 


\section{Coplanar spin textures}

Here we consider the case of graphene coupled to coplanar noncollinear spin textures, shown in Fig. 6. We address the weak-coupling limit $(J \ll t)$ at half filling. In the case of a purely in-plane exchange field, the Hamiltonian can be made real by a rotation onto the Pauli matrices $\sigma_{x}, \sigma_{z}$, leading to a vanishing Hall response. We show in Fig. 6 examples of band structures of triangular and rectangular graphene unit cells subjected to fully in-plane exchange field. Although a band gap opens, the calculated Berry curvature and thereby the Chern number vanish in both cases.

The necessity of a noncoplanar spin texture can be easily understood in terms of the symmetry properties of the Chern number. Without loss of generality, the exchange field of a coplanar spin texture can be expressed in terms of the Pauli matrices $\sigma_{x}$ and $\sigma_{z}$, provided the exchange field is rotated to lie in the $x z$ plane,

$$
\vec{M} \cdot \vec{\sigma}=M_{x} \sigma_{x}+M_{z} \sigma_{z},
$$

turning the exchange term of the Hamiltonian purely real, and therefore also the full Hamiltonian. Since the Chern number is odd under conjugation

$$
K: \mathcal{C} \rightarrow-\mathcal{C}
$$

and a purely real Hamiltonian is invariant under conjugation

$$
K: \mathcal{H} \rightarrow \mathcal{H}^{*}=\mathcal{H}
$$

the Chern number is identically zero $(\mathcal{C}=0)$ for a coplanar spin texture. Thus, noncoplanar spin arrangements are necessary to induce the anomalous Hall phase.

\section{Intervalley mixing}

In some instances the skyrmion lattice will be commensurate with the carbon honeycomb lattice, the two valleys in the band structure of graphene could be folded to a single point, allowing intervalley mixing. In particular, for the triangular lattice, the folding of the two valleys onto the $\Gamma$ point takes place for $3 n \times 3 n$ unit cells. For a rectangular unit cell, the folding takes place for a supercell $3 n$ in the zigzag direction. In these situations, intervalley scattering can open a trivial gap, as shown in Fig. 7, for such behavior. Interestingly, the Berry curvature can be nonzero, but the Chern number vanishes. Importantly, this situation requires a fine-tuning of the skyrmion and carbon lattices. In general, this is not the case, and nontrivial gaps are to be expected.
[1] F. D. M. Haldane, Phys. Rev. Lett. 61, 2015 (1988).

[2] C. L. Kane and E. J. Mele, Phys. Rev. Lett. 95, 226801 (2005).

[3] C. L. Kane and E. J. Mele, Phys. Rev. Lett. 95, 146802 (2005).

[4] D. A. Abanin, P. A. Lee, and L. S. Levitov, Phys. Rev. Lett. 96, 176803 (2006).

[5] A. F. Young, J. D. Sanchez-Yamagishi, B. Hunt, S. H. Choi, K. Watanabe, T. Taniguchi, R. C. Ashoori, and P. Jarillo-Herrero, Nature (London) 505, 528 (2014).

[6] K. S. Novoselov, Z. Jiang, Y. Zhang, S. V. Morozov, H. L. Stormer, U. Zeitler, J. C. Maan, G. S. Boebinger, P. Kim, and A. K. Geim, Science 315, 1379 (2007).

[7] Z. Qiao, S. A. Yang, W. Feng, W.-K. Tse, J. Ding, Yu. Yao, J. Wang, and Q. Niu, Phys. Rev. B 82, R161414 (2010).

[8] K. Von Klitzing, Rev. Mod. Phys. 58, 519 (1986).

[9] M. Konig, S. Wiedmann, C. Brüne, A. Roth, H. Buhmann, L. W. Molenkamp, X. L. Qi, and S. C. Zhang, Science 318, 766 (2007).

[10] P. W. Anderson and H. Hasegawa, Phys. Rev. 100, 675 (1955).

[11] D. Loss, P. Goldbart, and A. V. Balatsky, Phys. Rev. Lett. 65, 1655 (1990).

[12] E. Muller-Hartmann and E. Dagotto, Phys. Rev. B 54, R6819(R) (1996).

[13] J. Ye, Y. B. Kim, A. J. Millis, B. I. Shraiman, P. Majumdar, and Z. Tesanovic, Phys. Rev. Lett. 83, 3737 (1999).

[14] M. J. Calderon and L. Brey, Phys. Rev. B 63, 054421 (2001).

[15] I. Martin and C. D. Batista, Phys. Rev. Lett. 101, 156402 (2008).

[16] L. N. Bulaevskii, C. D. Batista, M. V. Mostovoy, and D. I. Khomskii, Phys. Rev. B 78, 024402 (2008).

[17] Y. Kato, I. Martin, and C. D. Batista, Phys. Rev. Lett. 105, 266405 (2010).
[18] N. Nagaosa, J. Sinova, S. Onoda, A. H. MacDonald, and N. P. Ong, Rev. Mod. Phys. 82, 1539 (2010).

[19] Y. Taguchi, Y. Oohara, H. Yoshizawa, N. Nagaosa, and Y. Tokura, Science 291, 2573 (2001).

[20] K. Ohgushi, S. Murakami, and N. Nagaosa, Phys. Rev. B 62, R6065 (2000).

[21] J. Brede, N. Atodiresei, V. Aciuc, M. Bazarnkik, A. Al-Zubi, S. Blugel, and S. Wiesendanger, Nat. Nanotechnol. 9, 1018 (2014).

[22] Q.-Z. Wang, X. Liu, H.-J. Zhang, N. Samarth, S.-C. Zhang, and C.-X. Liu, Phys. Rev. Lett. 113, 147201 (2014).

[23] N. Nagaosa and Y. Tokura, Nat. Nanotechnol. 8, 899 (2013).

[24] J. Sampaio, V. Cros, S. Rohart, A. Thiaville, and A. Fert, Nat. Nanotechnol. 8, 839 (2013).

[25] S. Heinze, K. von Bergmann, M. Menzel, J. Brede, A. Kubetzka, R. Wiesendanger, G. Bihlmayer, and S. Blugel, Nat. Phys. 7, 713 (2011).

[26] K. Hamamoto, M. Ezawa, and N. Nagaosa, arXiv:1504.06024.

[27] D. Xiao, M.-C. Chang, and Q. Niu, Rev. Mod. Phys. 82, 1959 (2010).

[28] D. J. Thouless, M. Kohmoto, M. P. Nightingale, and M. den Nijs, Phys. Rev. Lett. 49, 405 (1982).

[29] J. L. Lado, N. García, and J. Fernández-Rossier, arXiv: 1502.07112

[30] F. D. M. Haldane, Phys. Rev. Lett. 93, 206602 (2004).

[31] Z. Wang, C. Tang, R. Sachs, Y. Barlas, and J. Shi, Phys. Rev. Lett. 114, 016603 (2015).

[32] S. Seki, X. Z. Yu, S. Ishiwata, and Y. Tokura, Science 336, 198 (2012).

[33] Z. Qiao, W. Ren, H. Chen, L. Bellaiche, Z. Zhang, A. H. MacDonald, and Q. Niu, Phys. Rev. Lett. 112, 116404 (2014). 